\title{
Algebre non associative e sistemi differenziati di Riccati in un problema di Genetica.
}

\author{
Memoria di Giulio ANDReoli (a Napoli)
}

A Giovanni Sansone nel suo 70mo compleanno.

Snnto. - La genetica mendeliana, depurata da fatti "leatori e da eventuali catene di MARKoFr, è riproducibile - come schema matematico - da algebre non associative. Vi è tuttavia, fra queste, un caso eccezionale, trattato più estesamente in questa memoria.

Interpretando poi le strutture successive di una collettività mendeliana come vettori, questi soddisfano ad un'equazione vettoriale del tipo RICCATI.

\section{§ 1. Generalità. Schema della ricerca.}

Nelle applicazioni matematiche a fenomeni biologici o sociali, si presen. tano quasi sempre algebre associative, al più non commutative.

Se però si vuol avere uno schema analitico della evoluzione di una collettività, secondo lo schema di eredità mendeliana $\left({ }^{1}\right)$, è necessario abbandonare le algebre associative.

Nel caso discontinuo, seguendo le direttive di VolterRa, si trova un'al. gebra non associativa; e nel caso continuo un sistema di equazioni differenziali quadratiche, più generali di quelle di Rrcoatr.

Il caso che svilupperemo esplicitamente, è singolare, nel senso che il numero di certi parametri si abbassa, ed il sistema differenziale quadratico si riduce proprio ad uno di RICCATI.

Il legame intercedente fra l'algebra non associativa ed il sistema di Riccati è precisamente quello dovuto al concetto di integrazione nelle algebre, a sua volta estensione di quello analogo per le matrici introdotto dal Volterra e dallo Schlesinger.

Per seguire il colleganento fra i concetti matematici usati, e quelli di genetica, citiamo - oltre le classiche opere del Volterra - un gruppo di note nostre, sui sistemi differenziali di RrCCATI, comparse nei Rendiconti dell'Accademia delle Scienze di Napoli e nel Giornale di Battaglini, intorno

(1) Cfr. ad esempio, per le definizioni usate in biologia e per le teorie di Volterra. E. Padoa, Storia della vita sulla Terra, Feltrinelli, Milano, 1958, pag. 305 e seg.

T. H. Morgan, Embriologia e Genetica, Einaudi (Ed. scient.), 1958.

G. Montalenti, Elementi di Genetica, Cappelli, Bologna, 1939.

U. D'Ancona, La lotta per l'esistenza, Linaudi (Ed. scien.), 1942.

V. Volterra, La lutte pour l'existence, Gauthier-Villars, 
98 G. ANDREOLI: Algcbre non associative e sistemi differenziati di Riccati, cco.

al 1919-22, un'altro gruppo, proprio su questioni di genetica applicata a collettività e trattate matematicamente, ancora negli stessi Rendiconti, nello stesso periodo; ed infine un gruppo di tre memorie, gia comparse negli Atti della Accademia Gioenia di Catania e ristampate ora nel Giornale di Mat. di Battaglini (due di esse) e nella Ricerca (l'altra):

Dai rendiconti della $R$. Accademia di Scienze Fisiche e Matematiche di Napoli :

G. ANDREOLI: 1) Sistemi differenziali ad una variabile, Vol. XXV, 1919; 2) Sopra alcune proprietà di certi sistemi differenziali, Vol. XXVI, 1920; 3) Sui sistemi di epuazioni differenziali ordinarie, Vol. XXVII, 1921 ; 4) Sistemi differenziali di Riccati, Vol. XXVII, 1921; 5) Sui sistemi differenziali lineari, Vol. XXVII, 1921; 6) Sulle funzioni di composizione di watrici (funzioni isogene), 1934 (ristampato ne "La Ricerca", Anno, X, nº. 1, 1959).

Dagli atti dell'Accademia Gioenia di Catania:

G. ANDREOLI : Equazioni differenziali lineari, funzioni nelle algebre, operatori funzionali, Memorie I e II, 1932-33 (ristampato nel «Giornale di Matematiohe di Battaglini», Vol. LXXXVI. Fasc. I, 1958).

Per chiarire quanto svilupperemo in seguito conviene anzitutto rilevare che prescindiamo dalle fluttuazioni statistiche o probabilistiche; e che quindi introdurremo uno schema matematico «rigido». Inoltre verranno esaminati «universi» o «popolazioni o «collettività » o "insiemi» suddivisi in classi riguardo alle modalita di certi caratteri posseduti dagli "elementi» o «individui " singoli che li formano. Se la totalità è $U$, e le modalità del carattere $A$ sono $A^{(1)}, A^{(2)}, \ldots A^{(n)}$, diremo che il carattere $A^{(r)}$ è posseduto da $\alpha^{(r)}$ individui.

Si intende che le varie modalità di $A$ si escludono mutuamente nello stesso individuo; inoltre le classi verranno indicate con lo stesso simbolo della modalita posseduta. Siccome intendiamo seguire i rapporti fra le $A$, tralasceremo l'accrescimento di $U$ (nel caso generico e se non indicato diversamente) e considereremo la ripartizione proporzionale, sicchè risulterà :

$$
\alpha^{(1)}+\alpha^{(2)}+\ldots+\alpha^{(r)}=1 .
$$

Ammetteremo che da uno stadio si passi al successivo con due operazioni. La prima, di accoppiamento, che consiste nel formare delle coppie con due elementi dello siesso staủio.

La seconda, di generazione, che consiste nel passare da quello stadio ad uno successivo, sostituendo ad ogni coppia un certo insieme di nuovi individui delle diverse classi, diverso a seconda dell'appartenenza dei genitori alle varie classi di-coppie.

Questa sostituzione arviene secondo le leggi mendeliane.

Qui cade acconcio rilevare che la formazione delle coppie può, a sua volta, seguire due schemi; gli estremi sono $i$ seguenti. 
G. ANDrmour: Algebre non associative e sistemi differenziati di Riccati, ece. 99

O vi è un eriterio di selezione (omogamia, endogamia, esogamia - ristrette, complete o attenuate) oppure vi paò essere assoluta libertà e possibilità di accoppiamento.

Noi accetteremo quest' ultimo caso; il che conduce ad espressioni quadratiche.

In altri termini, supponiamo che avvengano tutti gli accoppiamenti pos. sibili fra gli $A^{(r)}$ e gli $A^{(s)}$, e, per le leggi della probabilitò, questi saranno in numero di $\alpha^{(r)} \alpha^{(s)}$, con $r=s$ or $r \neq s$.

Il fenomeno è netto e possibile in collettività viventi in ambiente ristretto, purchè costituite da individui aventi una mobilitz sufficiente per poter ricovrire tutto l'ambiente; ad esempio piante anemofile.

Con tali premesse, si vede che valgono almeno le seguenti possibilità, per la prima fase.

I. Collettività divise in generazioni nettamente staccate; ed allora

$$
U_{n+1}=U_{n}^{2}
$$

II. Collettività con sovrapposizione parziale di generazioni (ed esclusa la scomparsa di individui) :

$$
U_{n+1}=\rho_{n} U_{n}^{2}+\sigma_{n} U_{n}
$$

ove $\rho_{n}+\sigma_{n}=1, \rho_{n}$ e $\sigma_{n}>0$ (caso particolare $\rho$ e costanti).

III. Collettivita del tipo precedente con immissione di un'aliquota di altra collettività :

$$
U_{n+1}=\rho_{n} U_{n}^{2}+\sigma_{n} U_{n}+\tau_{n} V_{n} ; \quad\left(\rho_{n}+\sigma_{n}+\tau_{n}=1\right)
$$

e le analoghe ove si tratti di collettività ohe variano continuamente nel tempo. Per queste ultime valgono le equazioni differenziali simboliche di RICCATI :

IV. $u^{\prime}(t)=u^{2}(t)$

V. $u^{\prime}(t)=\rho(t) u^{2}(t)+\sigma(t) u(t)$

VI. $u^{\prime}(t)=\rho(t) u^{2}(t)+\sigma(t) u(t)+\theta(t) v(t)$.

Naturalmente queste equazioni vanno completate con la utilizzazione delle leggi genetiche; per le quali da

$$
U_{n} \times V_{n} \equiv \Sigma \alpha_{n}^{(r)} A^{(r)} \cdot \Sigma \beta_{n}^{(s)} A^{(s)}
$$

si passa a

$$
U_{n} \times V_{n}=W_{n}=\Sigma^{\prime} \gamma_{n}^{(t)} A^{(t)},
$$


$100 \mathrm{G}$. ANDREoLI: Algcbre won associative e sistemi aiffereneiati di Riccati, ecc.

dove le $\gamma^{(r)}$ sono bilineari nelle $\alpha^{(r)}, \beta^{(s)}$, tramite i coefficienti di struttura $c_{t r s}$, i quali - mancando l'associativita - non sono vincolati da altre equazioni che non siano quelle dovute alla commutatività. Lo stesso esempio che sviInppiamo nel $\$$ seguente, resta immutato sostanzialmente se si hanno ancora tre tipi $A, M, B$, ma cambiano i coefficienti nel prodotto: tralasciamo di effettuare gli ovvi sviluppi.

Il problema verra qui considerato in tre stadi divarsi. Dapprima tratteremo il caso di una collettività nella quale le varie e successive generazioni sieno del tutto staccate fra loro; come generalmente avviene per le piante annuali e per gli insetti.

Poi tratteremo il caso di generazioni che si sovrappongono parzialmente; quale può essere il caso di piante perenni o di animali a lunga vita, ma a periodo generativo unico (o al più doppio) per anno. Ed infine il caso di generazione continua, quale il caso di infusori o batteri o popolazioni umane.

Oltre questi tre casi, esamineremo che cosa avviene allorchè due popo. lazioni separate vengono a fondersi oppure entrano in coniugazione esogamica.

\section{\$ 2. Collettività a generazioni distinte.}

Consideriamo una certa collettivita $U$, nella quale valgano leggi di eredità $\left({ }^{*}\right)$ mendeliane; e prendiamo in esame la presenza di una coppia di caratteri - diciamo $a$ dominante, e $b$ recessivo.

Quindi nella popolazione $U$, vi saranno individui con il corredo cromosomico avente il genide $(a \alpha)$, altri con il genide $(a b)$, altri infine con il genide $(b b)$; e per trattare un easo più semplice, supporremo che i genotipi $(a a)$, $(a b),(b b)$ sieno discernibili fra loro e quindi sieno anche fenotipi; ad esempio $(a a)$ dia il carattere «rosso» $(b b)$, bianco ed $(a b)$ rosa.

Non ci ocoupiamo dei numeri assoluti di individni con i vari caratteri; sibbene solo delle loro proporzioni relative.

Danque, in $U$ vi sieno

$$
\begin{aligned}
\alpha \text { individui } & A \equiv(a, a) \\
\mu \quad 》 \quad M & \equiv(a, b) \\
\beta \quad 》 \quad B & \equiv(b, b)
\end{aligned}
$$

con $\alpha+\mu+\beta=1$, tutti positivi, ed indicanti rispettivamente le proporzioni con le quali gli $A$, gli $M$, i $B$ costituiscono $U$.

Tratteremo esaurientemente un caso, il più semplice dal punto di vista genetico.

Esso però, dal punto di vista matematico, rappresenta un caso di ecce-

(*) Vedi nota esplicativa, in fondo. 
G. ANDreour: Algebre non associative e sistemi differenziati di Riccati, ecc. 101

zione, sia perchè è ricavato dal caso generale dando particolari valori a certe costanti, sia perchè ̀े effettivamente singolare, nel senso che valgono particolari relazioni che non valgono, nè trovano analogia nel caso generale.

Eliminiamo l'influsso statistico; e supponiamo ohe gli individui di ambo i sessi sieno rappresentati nelle tre classi, in misura eguale; che la coniugazione e la proliferazione avvengano in misura direttamente proporzionali ad ambo le classi, e che vi sia un netto distacco fra una generazione e la successiva.

Talo è il caso di insetti a breve vita perfetta o di piante.

Quindi generati da coppie della prima classe, gli individni della nuora generazione saranno in numero proporzionale ad $\alpha^{2}$, quelli della prima con la seconda, in numero di $\mu a$ e cosi via.

Orbene, le leggi mendeliané pongono come norma che:

I. Il coniugio di due individai $(A A)$ dà esclusivamente prole $(A A)$; ciò in quanto - cromosomicamente - il nuovo individuo si forma con un cromosomo del primo ed uno del secondo genitore.

II. Il coniugio di $(a a)$ con $(a b)$ darà metà individui $(a a)$ e metà $(a b)$, per lo stesso motivo.

III. Il coningio di $(a a)$ con $(a b)$ dà tutti individui $M \equiv(a b)$.

IV. Il coniugio di $(a b)$ con $(a b)$ dà un quarto di $(a a)$, metà di $(a b)$, un quarto di $(b b)$.

V. Il coniugio di $(a b)$ con $(b b)$ darà metà individui $(a b)$ e metà $(b b)$.

VI. Il coniugio di $(b b)$ con $(b b)$ darà tutti individui $(b b)$.

In un caso più generale, I, III, $V$ restano invariati mentre II darà un'aliquota $\xi$ di individui $(a a)$ ed $\eta$ di $(a b) ; V$ darà un'aliquota $\tau$ di individui $(a b)$ ed $\omega$ di individui $(b b)$ ed infine IV un'aliquota $\frac{\xi}{2}$ di $(a a) \frac{\eta+\tau}{2}$ di $(a b)$ e $\frac{\omega}{2} \mathrm{di}(b b)$.

In modo ancora più generale queste ultime tre aliquote (a somma 1) possono essere sostituite da $\varphi, \psi \cdot \theta$, a somma ancora unitaria.

Pertanto se $U_{0}$ indica, per aliquote la composizione iniziale di $U$, cioè

$$
U_{0} \equiv \alpha_{0} A+\mu_{0} M+\beta_{0} B
$$

la generazione successiva sarà ottenuta con

$$
U_{1}=U_{0}^{2}=\alpha_{1} A+\mu_{1} M+\beta_{1} B,
$$


$102 \mathrm{G}$. ANDREoLt: Algebre non associative e sistemi differenziati di Riccati, ccc.

ed in generale:

$$
U_{n+1}=U_{n}^{2} \equiv \alpha_{n+1} A+\mu_{n+1} M+\beta_{n+1} B=\left(\alpha_{n} A+\mu_{n} M+\beta_{n} B\right)^{2} .
$$

Ma per quanto precede la tabella di moltiplicazione commutativa delle tre unità $A, M, B$ è

$$
\left\{\begin{array}{c}
A^{2}=A \\
M A=A M=\frac{1}{2} A+\frac{1}{2} M \\
B A=A B=M \\
M^{2}=\frac{1}{4} A+\frac{1}{2} M+\frac{1}{4} B \\
B M=M B=\frac{1}{2} M+\frac{1}{2} B \\
B^{2}=B .
\end{array}\right.
$$

Si vede subito che tale operazione binaria di prodotto non è associativa. Invero :

$$
\begin{aligned}
& (A A) M=A M=\frac{1}{2} A+\frac{1}{2} M \\
& A(A M)=A\left(\frac{1}{2} A+\frac{1}{2} M\right)=\frac{3}{4} A+\frac{1}{4} M
\end{aligned}
$$

Per la suddetta legge di moltiplicazione si deduce che da:

$$
\begin{gathered}
U_{0}^{2}=\left(\alpha_{0} A+\mu_{0} M+\beta_{0} B\right)^{2}= \\
=\alpha_{0}^{2} A^{2}+\mu_{0}^{2} M^{2}+\beta_{0}^{2} B^{2}+ \\
+2 \alpha_{0} \mu_{0} A M+2 \mu_{0} \beta_{0} M B+2 \alpha_{0} \beta_{0} A B
\end{gathered}
$$

si ottiene

$$
\alpha_{1} A+\mu_{1} M+\beta_{1} B
$$

con

$$
\left\{\begin{array}{l}
\alpha_{1}=\alpha_{0}^{2}+\frac{1}{4} \mu_{0}^{2}+2 \cdot \frac{1}{2} \alpha_{0} \mu_{0} \\
\mu_{1}=\frac{1}{2} \mu_{0}^{2}+2 \cdot \frac{1}{2} \alpha_{0} \mu_{0}+2 \cdot \frac{1}{2} \beta_{0} \mu_{0}+2 \alpha_{0} \beta_{0} \\
\beta_{1}=\beta_{0}^{2}+\frac{1}{4} \mu_{0}^{2}+2 \cdot \frac{1}{2} \beta_{0} \mu_{0}
\end{array}\right.
$$


G. ANDREoLI: Algebre non associative e sistemi differenziati di Riccati, ece. 103

orvero

$$
\left\{\begin{array}{l}
\alpha_{1}=\left(\alpha_{0}+\frac{1}{2} \mu_{0}\right)^{2} \\
\mu_{1}=2\left(\alpha_{0}+\frac{1}{2} \mu_{0}\right)\left(\beta_{0}+\frac{1}{2} \mu_{0}\right) \\
\beta_{1}=\left(\beta_{0}+\frac{1}{2} \mu_{0}\right)^{2}
\end{array}\right.
$$

Ed è subito visto che anche :

$$
\alpha_{1}+\mu_{1}+\beta_{1}=\left[\left(\alpha_{0}+\frac{1}{2} \mu_{0}\right)+\left(\beta_{0}+\frac{1}{2} \mu_{0}\right)\right]^{2}=1,
$$

e quindi sono anch' esse percentuali.

Quindi una qualsiasi generazione, diciamo $U_{n+1}$; si ottiene dalla precedente con la

e quindi con le:

$$
U_{n+1}=U_{n}^{2}
$$

$$
\left\{\begin{array}{l}
\alpha_{n+1}=\left(\alpha_{n}+\frac{1}{2} \mu_{n}\right)^{2} \\
\mu_{n+1}=2\left(\alpha_{n}+\frac{1}{2} \mu_{n}\right)\left(\beta_{n}+\frac{1}{2} \mu_{n}\right) \\
\beta_{n+1}=\left(\beta_{n}+\frac{1}{2} \mu_{n}\right)^{2}
\end{array}\right.
$$

Notiamo ora che la posizione $\alpha+\mu+\beta=1$ consente di eliminare $\mu$; in quanto $\mu=1-\alpha-\beta$.

Allora

$$
\begin{aligned}
& \alpha_{n}+\frac{1}{2} \mu_{n}=\frac{1+\alpha_{n}-\beta_{n}}{2} \\
& \beta_{n}+\frac{1}{2} \mu_{n}=\frac{1-\alpha_{n}+\beta_{n}}{2}
\end{aligned}
$$

ed introdotta la quantità

risulta

$$
\delta_{n}=\alpha_{n}-\beta_{n},
$$

$$
\left\{\begin{array}{l}
\alpha_{i+1}=\left(\frac{1+\delta_{n}}{2}\right)^{2} \\
\mu_{n+1}=\frac{1-\delta_{n}^{2}}{2} \\
\beta_{n+1}=\left(\frac{1-\delta_{n}}{2}\right)^{2}
\end{array}\right.
$$


104 G. Andreoli : Algebre non associative e sistemi differenziati di Riccati, ecc.

cio»

$$
\delta_{n+1}=\delta_{n}
$$

Cio implica che, a partire dalla seconda generazione, l'universo si ̀े «stabilizzato »: cioè

$$
U_{0} \neq U_{1} ; \quad U_{1}=U_{2}=U_{3}=\ldots .
$$

Osserviamo esplicitamente che tale stabilizzazione si ottiene solo nel caso speciale illustrato; $m$ nel caso generale (cfr. ultimo paragrafo) vi sono collettivita stabili verso cui tendono quelli in evolunione; ma in genere la stabilizzazione non si ottiene iterando un processo qualsiasi.

Se ricerchiamo quali sieno le popolazioni «stabili» (rispetto a quella coppia di caratteri, si intende), e che non mutano la loro composizione nei successivi stadi dovremo risolvere il sistema

$$
\left\{\begin{array}{l}
\alpha=\left(\alpha+\frac{\mu}{2}\right)^{2} \\
\mu=2\left(\alpha+\frac{\mu}{2}\right)\left(\beta+\frac{\mu}{2}\right) \\
\beta=\left(\beta+\frac{\mu}{2}\right)^{2} .
\end{array}\right.
$$

E logico che troveremo tutte le popolazioni di seconda generazione; ed avremo una semplice infinita di tipi, dipendenti da un parametro $\delta$ che varia $\mathrm{da}-1, \mathrm{a}+1$.

Invero, poniamo $\alpha-\beta=\delta ;$ è immediato che $\left(\alpha+\frac{\mu}{2}\right)-\left(\beta+\frac{\mu}{2}\right)=\delta$ ancora; e pertanto il sistema sovra scritto, diventa anche

$$
\left\{\begin{array}{l}
\left(\alpha+\frac{\mu}{2}\right)^{2}-\left(\alpha+\frac{\mu}{2}\right)+\frac{\mu}{2}=0 \\
\left(\beta+\frac{\mu}{2}\right)^{2}-\left(\beta+\frac{\mu}{2}\right)+\frac{\mu}{2}=0 \\
\left(\alpha+\frac{\mu}{2}\right)\left(\beta+\frac{\mu}{2}\right)=\frac{\mu}{2} .
\end{array}\right.
$$

In sostanza, $\left(\alpha+\frac{\mu}{2}\right)$ e $\left(\beta+\frac{\mu}{2}\right)$ sono le radici di

$$
z^{2}-z+\frac{\mu}{2}=0
$$

e la terza equazione è conseguenza di ciò. 
Allora,

$$
\delta=\alpha-\beta=\left(\alpha+\frac{\mu}{2}\right)-\left(\beta+\frac{\mu}{2}\right)
$$

si ottiene quale discriminante dell'equazione suddetta; ed eseguiti facili passaggi, si trova infine

$$
\begin{aligned}
& \alpha=\left(\frac{1+\delta}{2}\right)^{2} \\
& \beta=\left(\frac{1-\delta}{2}\right)^{2} \quad \delta \text { arbitrario, }-1 \leq \delta \leq 1 . \\
& \mu=\frac{1-\delta^{2}}{2}
\end{aligned}
$$

§ 3. Collettivita a generazioni intervallate e parzialmente sorrapposte.

Le ipotesi restino le stesse del caso precedente; senonchè in ogni stadio la popolazione è composta nelle proporzioni $\rho$ e $\sigma$ - che supporremo invariabili - dalla nuova generazione e dalla precedente; pertanto $\rho+\sigma=1$; e queste proporzioni $\rho$ e $\sigma$ sieno le stesse per $A, M, B$.

Avremo, ripetendo le stesse considerazioni di prima, che:

$$
\left\{\begin{array}{l}
\alpha_{n+1}=\rho\left(\alpha_{n}+\frac{\mu_{n}}{2}\right)^{2}+\sigma \alpha_{n} \\
\mu_{n+1}=2 \rho\left(\alpha_{n}+\frac{\mu_{n}}{2}\right)\left(\beta_{n}+\frac{\mu_{n}}{2}\right)+\sigma \mu_{n} \quad * \\
\beta_{n+1}=\rho\left(\beta_{n}+\frac{\mu_{n}}{2}\right)^{2}+\sigma \beta_{n} .
\end{array}\right.
$$

Simbolicamente :

$$
U_{n+1}=\rho U_{n}^{2}+\sigma U_{n} .
$$

Rileviamo subito che le popolazioni stabili, per il caso precedente, restano popolazioni stabili anche in questo caso; la semplice osservazione basta a dedurlo.

Tuttavia, per abundantiam, se la popolazione è stabile, sarà $\alpha_{n}=\alpha_{n+1}=\alpha$; $\mu_{n}=\mu_{n+1}=\mu ; \beta_{n}=\beta_{n+1}=\beta ;$ quindi

$$
\left\{\begin{array}{l}
\alpha=p\left(\alpha+\frac{\mu}{2}\right)^{2}+\sigma \alpha \\
\mu=2 \rho\left(\alpha+\frac{\mu}{2}\right)\left(\beta+\frac{\mu}{2}\right)+\sigma \mu \\
\beta=\rho\left(\beta+\frac{\mu}{2}\right)^{2}+\sigma \beta .
\end{array}\right.
$$


106 G. ANDREOLI: Algebre non associative e sistemi differenziati di Riccati, ecc.

Quindi $\alpha+\frac{\mu}{2}$ e $\beta+\frac{\mu}{2}$ sono le due radici di

$$
\rho z^{2}+(\sigma-1) z-(\sigma-1) \frac{\mu}{2}=0
$$

ed essendo $\rho=1-\sigma$, restano le radici di

$$
\rho\left[z^{2}-z+\frac{\mu}{2}\right]=0
$$

ehe è la stessa di prima, essendo $\rho \neq 0$, come è evidente.

Si rilevi ohe posto

$$
\delta_{n+1}=\alpha_{n+1}-\beta_{n+1}=\left(\alpha_{n+1}+\frac{\mu_{n+1}}{2}\right)-\left(\beta_{n+1}+\frac{\mu_{n+1}}{2}\right)
$$

risulta anche adesso

$$
\delta_{n+1}=\delta_{n}=\ldots=\delta_{0}
$$

come si vede sottraendo la prima e terza delle $*$, tenuto conto di $\alpha+\mu+\beta=1$, e di $\rho+\sigma=1$.

A lor volta le $*$ si possono anche scrivere:

$$
\left\{\begin{array}{l}
\alpha_{n+1}=\rho\left(\frac{1+\delta_{n}}{2}\right)^{2}+\sigma \alpha_{n} \\
\mu_{n-1}=\rho \frac{1-\delta_{n}^{2}}{2}+\sigma \mu_{n} \\
\beta_{n+1}=\rho\left(\frac{1-\delta_{n}}{2}\right)^{2}+\sigma \beta_{n}
\end{array}\right.
$$

ed iterando queste relazioni si giunge a

$$
\left\{\begin{array}{l}
\alpha_{n+1}=\rho\left(\frac{1+\delta_{0}}{2}\right)^{2}\left(1+\sigma+\ldots+\sigma^{n}\right)+\sigma^{n+1} \alpha_{0} \\
\mu_{n+1}=\rho\left(\frac{1-\delta_{0}^{2}}{2}\right)^{2}\left(1+\sigma+\ldots+\sigma^{n}\right)+\sigma^{n+1} \mu_{0} \\
\beta_{n+1}=\rho\left(\frac{1-\delta_{0}}{2}\right)^{2}\left(1+\sigma+\ldots+\sigma^{n}\right)+\sigma^{n+1} \beta_{0} .
\end{array}\right.
$$

Tenuto conto della $\rho+\sigma=1$, queste formale diventano, in definitiva:

$$
\left\{\begin{array}{l}
\alpha_{n+1}=\left(\frac{1+\delta_{0}}{2}\right)^{2}\left(1-\sigma^{n+1}\right)+\sigma^{n+1} \alpha_{0} \\
\mu_{n+1}=\frac{1-\delta_{0}^{2}}{2}\left(1-\sigma^{n+1}\right)+\sigma^{n+1} \mu_{0} \\
\beta_{n+1}=\left(\frac{1-\delta_{0}}{2}\right)^{2}\left(1-\sigma^{n+1}\right)+\sigma^{n+1} \beta_{0} .
\end{array}\right.
$$


G. ANDreoLI: Algebre non associative e sistemi differenziati di Riccati, ecc 107

Pertanto all'aumentare del numero d'ordine dello stadio, la popolazione tende a quella stabile che competerebbe alla originaria, se non ci fosse mesco. lanza fra due generazioni consecutive.

Come rappresentazione geometrica, si rilevi che tutte le popolazioni giacciono - come punti rappresentativi $x=\alpha, y=\mu, z=\beta-$ sul piano $\pi$ $x+y+z=1$, nel primo ottante.

Partendo da un punto qualunque $P$, di questo piano, le successive popolazioni vengono rappresentate da punti $P_{1}, P_{2}, P_{3} \ldots$ per i quali $x_{0}-z_{0}=$ $=x_{1}-z_{1}=x_{2}-z_{2}=\ldots ;$ quindi sulla intersezione con $\pi$ del piano $x-z=\delta$ passante per $P_{0}$ e sulla retta intersezione scorrono in progressione geometrica,

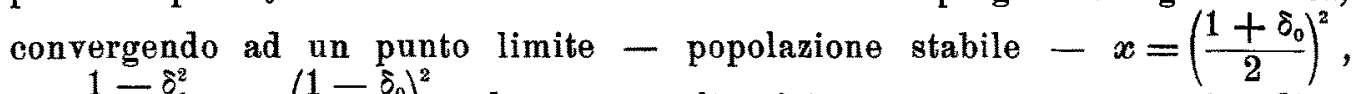
$y=\frac{1-\delta_{0}^{2}}{2}, z=\left(\frac{1-\delta_{0}}{2}\right)^{2} ;$ che a sua volta si trova su una certa conica di $\pi$ rappresentativa di tutie le popolazioni stabili.

\section{\& 4. L'evoluzione continua.}

Passiamo al caso nel quale le generazioni si continuano nel tempo ininterrottamente, senza "scatti»; quale ad esempio in forme inferiori di vita.

Le leggi di trasformazione continuano a valere; ma, allora $\alpha, \mu, \beta$ non saranno più soggette ad avere per somma l'unità; e se ammettiamo che l'eli. minazione agisca egualmente sui tipi $A, M, B$, cosi come il coniugio e la natalità, avremo - per un intervallo $\Delta t$, cui corrispondono le composizioni $U\left(t_{n+1}\right)$ finale, ed $U\left(t_{n}\right)$ iniziale - la legge differenziale

$$
\frac{U\left(t_{n+1}\right)-U\left(t_{n}\right)}{t_{n+1}-t_{n}}=k U^{2}\left(t_{n}\right)+l U(t) .
$$

La $k$ può essere assunta quale valore fisso in un primo esame, o dipendere dal tempo, in una ulteriore generalizzazione.

Il passaggio al limite porterà dunque a :

$$
\begin{gathered}
\alpha^{\prime}(t) A+\mu^{\prime}(t) M+\beta^{\prime}(t) B= \\
=k(t)\left\{\left(\alpha+\frac{1}{2} \mu\right)^{2} A+2\left(\alpha+\frac{1}{2} \mu\right)\left(\beta+\frac{1}{2} \mu\right) M+\left(\beta+\frac{1}{2} \mu\right)^{2} B\right\}+ \\
+l(t)\{\alpha A+\mu M+\beta B\} .
\end{gathered}
$$

Quindi l'evoluzione della collettività sara retta dal sistema differenziale

$$
\left.\begin{array}{l}
\alpha^{\prime}=k(t)\left(\alpha+\frac{1}{2} \mu\right)^{2}+l(t) \alpha(t) \\
\mu^{\prime}=2 k(t)\left(\alpha+\frac{1}{2} \mu\right)\left(\beta+\frac{1}{2} \mu\right)+l(t) \mu(t) \\
\beta^{\prime}=k(t)\left(\beta+\frac{1}{2} \mu\right)^{2}+l(t) \beta(t) .
\end{array}\right\} S
$$


$108 \mathrm{G}$. ANDREOLI: Algebre non associative e sistemi differenziati di Riccati, ece.

Questo è un sistema quadratico del tipo di quelli trovati dal VoLTERRA; ed è analogo ai sistemi di RICCATI.

Intanto se si pone

$$
\alpha(t)+\mu(t)+\beta(t)=u(t)
$$

si ricava subito:

$$
u^{\prime}(t)=k(t) u^{2}(t)+l(t) u(t)
$$

che è proprio un'equazione di Rrccatr.

Se $k, l$, sono costanti, allora

$$
\frac{d u}{(k u+l) u}=d t
$$

e l'integrale di questa dà la ben nota curva logistica, avente due assintoti (paralleli all'asse $t$ ) entro la cui striscia la curva si svolge.

Nel caso generale l'equazione di Rrocati, mancando del termine indipendente da $u$, diventa una di BerNodlur e riducibile alle quadrature.

Dal sistema $S$ possiamo dedurre un sistema di Rrccatr.

Poniamo :

$$
\alpha+\frac{1}{2} \mu=\xi ; \quad \beta+\frac{1}{2} \mu=\eta
$$

Risulta subito

$$
\left\{\begin{array}{l}
\xi^{\prime}(t)=k(t) \xi(t)\{\xi+\eta\}+l(t) \xi(t) \\
\eta^{\prime}(t)=k(t) \eta(t)\{\xi+\eta\}+l(t) \eta(t)
\end{array}\right.
$$

e questo è proprio un sistema di RIOCATI (*), in quanto la sua parte quadratica consta di un fattore lineare fisso per tutte le equazioni del sistema, moltiplicata per quell'incognita che figura a sinistra derivata; e la sua parte lineare varia da equazione ad equazione. Il sistema di RICCATI è riducibile perchè omogeneo.

Per la sua struttura, una qualsiasi combinazione lineare a coefficienti costanti, continua a soddisfare ad equazioni analoghe.

In particolare, questo e il caso di $\xi+\eta$, che risulta eguale ad $u$, come già visto; e di $\xi-\eta$ che risulta eguale a $\delta=\alpha-\beta$.

Vi ̀̀ da rilevare che il rapporto $\rho=\frac{\xi}{\eta}$ è costante.

Ed invero:

$$
\rho^{\prime}=\left(\frac{\xi}{\eta}\right)^{\prime}=\frac{\xi^{\prime} \eta-\xi \eta^{\prime}}{\eta^{2}} \text {. }
$$

$\mathrm{Ma}$ il numeratore si annulla, in quanto

$$
\xi^{\prime} \eta=\xi \eta^{\prime}=k \xi \eta\{\xi+\eta\}+l \xi \eta,
$$

dunque $\rho^{\prime}=0$ e $\rho=$ costante. 
G. ANDreoli: Algebre non associative e sistemi differenziati di Riccati, ecc. 109

Di conseguenza anche il rapporto fra $\xi-\eta=\alpha-\beta$ e $\xi+\eta=\alpha+\mu+$ $+\beta=u$ è costante.

Il sistema differenziale si riduce allora a :

$$
\left\{\begin{array}{l}
u^{\prime}(t)=l(t) u^{2}(t)+l(t) u(t) \\
\delta^{\prime}(t)=\{k(t) u(t)+l(t)\} \delta(t) \\
\xi^{\prime}(t)=\{k(t) u(t)+l(t)\} \xi(t) \\
\eta^{\prime}(t)=\{k(t) u(t)+l(t)\} \eta(t)
\end{array}\right.
$$

resta pertanto

$$
\frac{u^{\prime}(t)}{u(t)}=\frac{\delta^{\prime}(t)}{\delta(t)}=\frac{\xi^{\prime}(t)}{\xi(t)}=\frac{\eta^{\prime}(t)}{\eta(t)}=k(t) u(t)+l(t)
$$

che confermano, appunto, la proporzionalità costante di $\delta, \xi, \eta$ con $u$.

Ma avuti $\xi, \eta$ restano determinati $\alpha, \mu, \beta$ tramite equazioni lineari differenziali complete; ad esempio:

$$
\mu^{\prime}=2 k \xi \eta-l \mu
$$

darà $\mu ;$ e $\xi-\frac{\mu}{2}=\alpha, \eta-\frac{\mu}{2}=\beta$.

\section{§ 5. Mescolanza di due popolazioni ; afflusso continuo dall' esterno.}

Si abbiano adesso due popolazioni diverse $U^{\prime}, U^{\prime \prime}$ di composizione $\alpha^{\prime}, \mu^{\prime}$, $\beta^{\prime} ; \alpha^{\prime \prime}, \mu^{\prime \prime}, \beta^{\prime \prime} ;$ non stabilizzatesi e sieno mescolate nella proporzione reciproca $\rho, \sigma(\operatorname{con} \rho+\sigma=1)$.

La prima, porta alla stabilizzazione:

$$
\alpha_{1}^{\prime}=\left(\frac{1+\delta^{\prime}}{2}\right)^{2} ; \quad \mu_{1}^{\prime}=\frac{1-\delta^{\prime 2}}{2} ; \quad \beta_{1}{ }^{\prime}=\left(\frac{1-\delta^{\prime}}{2}\right)^{2}
$$

e la seconda analogamente.

La loro mescolanza crea una popolazione con

$$
\alpha=\rho \alpha^{\prime}+\sigma \alpha^{\prime \prime} ; \quad \mu=\rho \mu^{\prime}+\sigma \mu^{\prime \prime} ; \quad \beta=\rho \beta^{\prime}+\sigma \beta^{\prime \prime}
$$

e quindi, dall' essere :

$$
\delta=\rho \delta^{\prime}+\sigma \delta^{\prime \prime}
$$

si trae che la mescolanza si stabilizzerà con

$$
\alpha=\left(\frac{1+\delta}{2}\right)^{2} ; \quad \mu=\frac{1-\delta^{2}}{2} ; \quad \beta=\left(\frac{1-\delta}{2}\right)^{2} .
$$


110 G. ANDREoLI: Algebre non associative e sistemi differenziati di Riccati, ecc.

Nel caso di apporto nuovo, ad ogni generazione, le equazioni si modificano con un termine additivo,

$$
\begin{aligned}
& \alpha_{n+1}=\rho\left(\frac{1+\delta_{n}}{2}\right)^{2}+\sigma \alpha_{n}+\varphi_{n} \\
& \mu_{n+1}=\rho \frac{1-\delta_{n}^{2}}{2}+\sigma \mu_{n}+\varepsilon_{n} \\
& \beta_{n+1}=\rho\left(\frac{1-\delta_{n}}{2}\right)^{2}+\sigma \beta_{n}+\psi_{n}
\end{aligned}
$$

dove

$$
\rho+\sigma+\left(\varphi_{n}+\varepsilon_{n}+\psi_{n}\right)=s \text {. }
$$

Nel caso particolare che l'afflusso sia di tipo costante - e percio $\varepsilon, \varphi, \psi$ non dipendano da $n$, le formule si semplificano.

Intanto resta, nel caso generale,

$$
\delta_{n+1}=(\rho+\sigma) \delta_{n}+\left(\varphi_{n}-\psi_{n}\right)
$$

e posto $\varphi_{n}-\psi_{n}=\theta_{n}, \operatorname{con} \rho+\sigma=\tau$

$$
\delta_{n+1}=\tau \delta_{n}+\theta_{n}
$$

da cui :

$$
\delta_{n+1}=\theta_{n}+\tau \theta_{n-1}+\tau^{2} \theta_{n-2}+\ldots+\tau^{n} \theta_{0} .
$$

Nel caso di $\theta$ costante (che è quindi più ampio di $\varepsilon, \varphi, \psi$ costanti) sỉ ha semplicemente :

$$
\delta_{n+1}=\left(1+\tau+\ldots+\tau^{n}\right) \theta .
$$

Occorre tuttavia rilevare che due diverse generazioni originarie possono dar luogo ad una stessa prima generazione (e quindi stabile). In effetti il solo parametro essenziale è $\delta$; quindi due popolazioni con lo stesso $\delta$ daranno luogo alla stessa generazione seguente.

Quindi posto

$$
\begin{aligned}
& U_{0}=\alpha_{0} A+\mu_{0} M+\beta_{0} B ; \quad \alpha_{0}+\mu_{0}+\beta_{0}=1 \\
& V_{0}=\left(\alpha_{0}+k\right) A+\left(\mu_{0}-2 k\right) M+\left(\beta_{0}+k\right) B
\end{aligned}
$$

la sola condizione di possibilità è che:

$$
-\alpha_{0}-\beta_{0}<k<\frac{\mu_{0}}{2} .
$$


G. ANDREoLI : Algebre non associative e sistemi differenziati di Riccati, ecc. 111

Soddisfatta questa sard

$$
U_{1}=V_{1}
$$

La mescolanza $\rho U_{0}+\sigma V_{0}$ (con $\left.\rho+\sigma=1\right)$, conduce ad una popolazione

$$
W_{0} \equiv\left(\alpha_{0}+\sigma k\right) A+\left(\mu_{0}-2 \sigma k\right) M+\left(\beta_{0}+\sigma k\right) B,
$$

che conserva ancora lo stesso $\delta$.

Quindi l'influsso della mescolanza è effettivo solo in presenza di due diversi $\delta$.

A sua volta, nel caso di afflusso e di evoluzione continua, il sistema differenziale diventa :

$$
\left\{\begin{array}{l}
\alpha^{\prime}(t)=k(t)\left(\alpha+\frac{1}{2} \mu\right)^{2}+l(t) \alpha(t)+m(t) \\
\mu^{\prime}(t)=2 k(t)\left(\alpha+\frac{1}{2} \mu\right)\left(\beta+\frac{1}{2} \mu\right)+l(t) \mu+n(t) \\
\beta^{\prime}(t)=k(t)\left(\beta+\frac{1}{2} \mu\right)^{2}+l(t) \beta+p(t)
\end{array}\right.
$$

e posto

$$
\alpha+\beta+\mu=u(t) ; \quad m+n+p=g(t)
$$

sarà ancora :

$$
u^{\prime}(t)=k(t) u^{2}+l(t) u+g(t)
$$

che è un' equazione completa di Riccati.

Infine, se avessimo due popolazioni

$$
U^{\prime}=a^{\prime} A+\mu^{\prime} M+\beta^{\prime} B ; \quad U^{\prime \prime}=\alpha^{\prime \prime} A+\mu^{\prime \prime} M+\beta^{\prime \prime} B
$$

ed evitando l'omogamia (ciò̀ il coniugio di elementi di $U^{\prime}$ con altri anche di $U^{\prime}$ e di $U^{\prime \prime}$ con altri anche di $U^{\prime \prime}$ ) avendo esclusivamente esogamia; avremmo

$$
\begin{aligned}
U^{*} & =U^{\prime} \times U^{\prime \prime}=\left(\alpha^{\prime} A+\mu^{\prime} M+\beta^{\prime} B\right)\left(\alpha^{\prime \prime} A+\mu^{\prime \prime} M+\beta^{\prime \prime} B\right) \\
& =\alpha^{*} A+\mu^{*} M+\beta^{*} B
\end{aligned}
$$

troveremmo :

$$
\left\{\begin{array}{l}
\alpha^{*}=\left(\alpha^{\prime}+\frac{1}{2} \mu^{\prime}\right)\left(\alpha^{\prime \prime}+\frac{1}{2} \mu^{\prime \prime}\right) \\
\mu^{*}=\left(\alpha^{\prime}+\frac{1}{2} \mu^{\prime}\right)\left(\beta^{\prime \prime}+\frac{1}{2} \mu^{\prime \prime}\right)+\left(\alpha^{\prime \prime}+\frac{1}{2} \mu^{\prime \prime}\right)\left(\beta^{\prime}+\frac{1}{2} \mu^{\prime}\right) \\
\beta^{*}=\left(\beta^{\prime}+\frac{1}{2} \mu^{\prime}\right)\left(\beta^{\prime \prime}+\frac{1}{2} \mu^{\prime \prime}\right)
\end{array}\right.
$$


112 G. Andreou : Algebre non associative o sistemi differenziati di Riccati, ecc.

\section{§ 6. Impostazione nel caso generale.}

Supponiamo ora di avere la possibilità di genidi multipli - e corredo cromosomico metà.

Sa $n$ genidi eguali, vi saranno le combinazioni $n$ tutti $a ;(n-1)$ di $a$ ed un $b ; \ldots$ tutti $n$ sono $b$.

Caratterizziamoli con il numero delle $a$ presenti.

Dunque, nei casi sinora trattati, si aveva soltanto (1) o (0), come simbolo; mentre se $n=2$, vi sono i simboli (2), (1), (0).

Il corredo oromosomico normale consta di una combinazione di due cor. redi semplici; quindi avremo $\frac{1}{2} n(n+1)$ casi possibili, che denoteremo con $(r, s)$.

Il coniugio fra due gameti e quindi dato da

$$
(r, s) \times(0, \sigma)
$$

e spezzandosi ognuno in un componente di una coppia $\theta$ in un componente dell' altra coppia, avremo come generazione seguente le possibilità

$$
(r, \rho) ; \quad(r, \sigma) ; \quad(s, \rho) ; \quad(s, \sigma) ;
$$

ed a seconda delle coincidenze dei diversi valori, alcuni dei discendenti potranno coincidere.

Vi sono le seguenti classi di possibilità:

I. Quattro simboli numerici tutti diversi; ed allora il risultato del prodotto è la somma di quattro addendi tutti diversi con coefficienti $\frac{1}{4}$.

II. Due simboli eguali, e due diversi; con le sottoclasssi:

II'. I simboli eguali nella stessa parentesi

$$
(r, r) \times(\rho, \sigma) .
$$

Allora il risultato del prodotto è

$$
(r, p),(r, \sigma)
$$

con coefficienti $\frac{1}{2}$.

II". I simboli eguali in parentesi diverse

$$
(r \rho) \times(r \sigma)
$$

Si hanno ancora quattro gruppi con coefficiente $\frac{1}{4}$. 
G. ANDREolI: Algebre non associative e sistemi differenziati di Riccati, ecc. 113

III. Una coppia di simboli eguali fra loro, ed un'altra eguale fra loro; colle sottoclassi:

III'. Nelle stesse parentesi l' eguaglianza

$$
(r, r) \times(s, s)
$$

si hanno, come risultati, solo

$$
(r, s)
$$

con coefficiente 1.

III". Due parentesi eguali

$$
(r, s) \times(r, s)
$$

si ha, come risultato

$$
(r, r),(r, s),(s, s)
$$

con coefficienti rispettivi $\frac{1}{4}, \frac{1}{2}, \frac{1}{4}$.

IV. Tre simboli eguali fra loro ed uno diverso.

$$
(r, r) \times(r, s)
$$

ed il risultato è soltanto

$$
(r, r),(r, s)
$$

con coefficienti $\frac{1}{2}$.

\section{Quattro simboli tutti eguali}

$$
(r, r)(r, r)
$$

con risultato solo $(r, r)$ ancora.

E quindi facile stabilire la tabella di moltiplicazione delle « unità » di questa algebra non associativa.

Come prima si hanno $i$ tre casi retti sempre rispettivamente da:

$$
\begin{aligned}
& U_{n+1}=U_{n}^{2} \\
& U_{n+1}=\lambda U_{n}^{2}+\mu U_{n} \quad(\lambda+\mu=1) \\
& u^{\prime}(t)=\lambda u^{2}(t)+\mu u(t)
\end{aligned}
$$

scritture simboliche abbreviate per:

$$
\begin{aligned}
& U_{n}=\Sigma \alpha_{(r, s)}^{(n)} A_{(r, s)} \\
& u(t)=\Sigma \alpha_{r, s}(t) \cdot A_{(r, s)},
\end{aligned}
$$


114 G. ANDREoLI : Algebre non associntive e sistemi differenziati di Riccati, ecc.

dove a sua volta $A_{(r, s)}=A_{(s, r)}$ sta per i simboli indicati con $(r, s)$ appunto; $r, s=0, \ldots, n$. RICCAIII

Si giunge sempre ad una trasformazione quadratica e ad un sistema di

Se $\lambda$ e $\mu$ sono costanti, questo è riducibile alle quadrature con funzioni elementari ; e la discussione è agevole.

Altrimenti d̀ solo possibile dare formule di maggiorazione.

Nel caso di soli tre tipi, $A, M, B$, con la tabella di moltiplicazione generale

$$
\left\{\begin{array}{l}
A^{2}=A ; \quad B^{2}=B ; \quad A B=M \\
A M=\xi A+\eta M \\
M^{2}=\varphi A+\psi M+\theta B \\
M B=\tau M+\omega B .
\end{array}\right.
$$

Si vede che il passaggio (per generazioni staccate) dall' una alla successiva, con

$$
U_{n}=\alpha_{n} A+\mu_{n} M+\beta_{n} B
$$

avviene ponendo:

$$
\begin{aligned}
& \alpha_{n+1}=\alpha_{n}^{2}+2 \xi \alpha_{n} \mu_{n}+\varphi \mu^{2} \\
& \mu_{n+1}=\psi \mu_{n}^{2}+2 \eta \alpha_{n} \mu_{n}+2 \tau \beta_{n} \mu_{n}+2 \alpha_{n} \beta_{n} \\
& \beta_{n+1}=\beta_{n}^{2}+2 \omega \beta_{n} \mu_{n}+\theta \mu^{2} .
\end{aligned}
$$

Si vede che, essendo $\xi+\eta=\tau+\omega=\varphi+\psi+\theta=1$, ed $\alpha_{n}+\mu_{n}+\beta_{n}=1$ sarà anche $\alpha_{n+1}+\beta_{n+1}+\mu_{n+1}=1$.

Ove risulti, in particolare

$$
\begin{aligned}
& \varphi=\xi^{2} \\
& \theta=\omega^{2}
\end{aligned}
$$

sard̀ anche di conseguenza :

$$
\begin{aligned}
& \alpha_{n+1}=\left(\alpha_{n}+\xi \mu_{n}\right)^{2} ; \quad \beta_{n+1}=\left(\beta_{n}+\omega \mu_{n}\right)^{2} \\
& \mu_{n+1}=2\left(\alpha_{n}+\xi \mu_{n}\right)\left(\beta_{n}+\omega \mu_{n}\right)
\end{aligned}
$$

essendo

$$
\alpha_{n+1}+\mu_{n+1}+\beta_{n+1}=1=\left[\left(\alpha_{n}+\xi \mu_{n}\right)+\left(\beta_{n}+\omega \mu_{n}\right)\right]^{2} ;
$$

ma questo implica, entro parentesi quadra, anche $\xi+\omega=1$; ovvero $\omega=\eta$ e quindi $\xi=\tau$.

Con questa riduzione, per $\mu_{n+1}$ si avrà identicamente:

$$
2\left(\alpha_{n}+\xi \mu_{n}\right)\left(\beta_{n}+\omega \mu_{n}\right)=\psi \mu_{n}^{2}+2 \eta \alpha_{n} \mu_{n}+2 \tau \beta_{n} \mu_{n}+2 \alpha_{n} \beta_{n}
$$


G. ANDREOLI : Algebre non associative e sistemi differenziati di Riccati. ecc. 115

e quindi necessario che sia

$$
\omega \xi=\psi
$$

oltre che $\omega=\eta$ e $\tau=\xi$ come gia visto.

Pertanto, in questo caso speciale, le relazioni fondamentali dell'algebra diventano

$$
\left\{\begin{array}{l}
A^{2}=A ; \quad B^{2}=B ; \quad A B=M \\
A M=\xi A+\eta M ; \quad B M=\xi M+\eta B \\
M^{2}=\xi^{2} A+2 \xi \eta M+\eta^{2} B
\end{array}\right.
$$

ed il passaggio da uno stadio al successivo, ciò̀

$$
U_{n+1}=U_{n}^{2}
$$

è dato da

$$
\left\{\begin{array}{l}
\alpha_{n+1}=\left(\alpha_{n}+\xi \mu_{n}\right)^{2} \\
\mu_{n+1}=2\left(\alpha_{n}+\xi \mu_{n}\right) \cdot\left(\beta_{n}+\eta \mu_{n}\right) \\
\beta_{n+1}=\left(\beta_{n}+\eta \mu_{n}\right)^{2}
\end{array}\right.
$$

e per $\xi=\eta=\frac{1}{2}$ si ritrova il caso originariamente sviluppato.

Come invariante, non è più il $\delta$ che giuoca: anzi non ve ne è alcuno.

La ricerca delle collettivitì stabili, in questo caso (meno ampio del generale) si riconduce al sistema:

$$
\left\{\begin{array}{l}
\alpha=(\alpha+\xi \mu)^{2} \\
\mu=2(\alpha+\xi \mu)(\beta+\eta \mu) \\
\beta=(\beta+\eta \mu)^{2}
\end{array}\right.
$$

e quindi - tenute presenti la prima ed ultima equazione, a

$$
\left\{\begin{array}{l}
(\alpha+\xi \mu)^{2}-(\alpha+\xi \mu)+\xi \mu=0 \\
(\beta+\eta \mu)^{2}-(\beta+\eta \mu)+\eta \mu=0
\end{array}\right.
$$

Dunque

$$
\begin{aligned}
\alpha+\xi \mu & =\frac{1 \pm \sqrt{1-4 \xi^{2} \mu^{2}}}{2} \\
\beta+\mu \eta & =\frac{1 \pm \sqrt{1-4 \eta^{2} \mu^{2}}}{2} .
\end{aligned}
$$

Queste, sommate, danno

$$
\alpha+(\xi+\eta) \mu+\beta+\frac{2 \pm \sqrt{1-4 \xi^{2} \mu^{2}} \pm \sqrt{1-4 \eta^{2} \mu^{2}}}{2} ;
$$


116 G. ANDReLI: Algebre non associative e sistemi differenziati di Riccati, ecc.

ed essendo $\xi+\eta=1 ; \alpha+\mu+\beta=1$, resta

$$
1=\frac{2 \pm \sqrt{1-4 \xi^{2} \mu^{2}} \pm \sqrt{1-4 \eta^{2} \mu^{2}}}{2}
$$

e pertanto

$$
\sqrt{1-4 \xi^{2} \mu^{2}}=\sqrt{1-4 \eta^{2} \mu^{2}}
$$

che infine porta a

$$
\xi^{2} \mu^{2}=\eta^{2} \mu^{2}
$$

Escluso il caso particolare (e singolare) $\xi=\eta=\frac{1}{2}$, resta solo $\mu=0$ possibile.

Ma allora, per $\alpha$ e $\beta$ si hanno le equazioni

$$
\alpha^{2}-\alpha=0 ; \quad \beta^{2}-\beta=0 ; \quad \alpha+\beta=1 .
$$

Quindi $o \alpha=0, \beta=1 ; 0 \alpha=1 \in \beta=0$ come sole possibilità.

Cio permette di dedurre che, a seconda delle condizioni iniziali, una qualsiasi popolazione evolventesi non può che tendere ad una forma "pura» eliminando gli ibridi - se tende ad un limite: oppure oscillare indefinita. mente: salvo a uon essere nel caso singolare $\xi=\eta=\frac{1}{2}$, come già visto.

Comunque - in ogni caso - resta stabilito che :

I. Uno schema genetico, sul tipo di eredita mendeliane conduce sem. pre ad un'algebra non associativa.

II. Le equazioni che reggono i vari tipi di collettività in evoluzione, a stadi parzialmente mescolati, e con afflusso di popolazioni immigrate, sono

$$
D_{n+1}=p_{n} U_{n}^{2}+q_{n} U_{n}+V_{n},
$$

III. Quelle che invece reggono l'evoluzione continua sotto forma differenziale, sono :

$$
U^{\prime}(t)=p(t) U^{2}(t)+q(t) U(t)+V(t)
$$

e quindi analoghi a sistemi differenziali di RICCATI-Volterra.

IV. Le $U$ si presentano sotto la forma di elementi di un'algebra:

$$
U \equiv \Sigma \alpha_{,} A_{r}
$$

dove le $A$, soddisfano alla proprietả commutativa del prodotto, ma non a quella associativa; e quindi

$$
A_{r} \cdot A_{s}=\Sigma c_{r s t} A_{t}
$$

